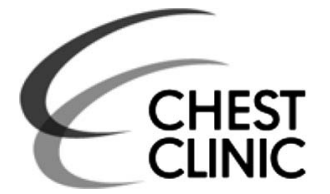

CASE BASED DISCUSSION

\title{
A case for the therapeutic use of perfluorocarbon in pulmonary atelectasis
}

\author{
Shreya Bali, ${ }_{1}$ Gareth Morgan, ${ }^{2}$ Andrew Nyman, ${ }^{3}$ Simona Turcu, ${ }^{4}$ Andrew Durward ${ }^{3}$
}

'Department of Anaesthesia, Homerton University Hospital, London, UK

${ }^{2}$ Department of Paediatric

Cardiology, Evelina Children's Hospital, London, UK

${ }^{3}$ Paediatric Intensive Care Unit, Evelina Children's Hospital, London, UK

${ }^{4}$ Department of Respiratory Medicine, Evelina Children's Hospital, London, UK

\section{Correspondence to}

Dr Andrew Durward, Paediatric Intensive Care Unit, Evelina Children's Hospital, Westminister Rd, London SE1 7EH, UK; Andrew.Durward@ gstt.nhs.uk

Received 21 March 2016 Revised 10 November 2016 Accepted 19 November 2016 Published Online First 20 December 2016
This is a case report formatted as a clinical handover discussion between a junior ward paediatrician (SB) and senior paediatric intensive care consultant (AD) and cardiology consultant (GM).

SB: I have been handed over a 3-year-old patient from the paediatric intensive care unit (PICU). She has a complex medical history and spent the first year of her life in PICU. She had a repair of a hypoplastic-aortic-arch and a tracheo-oesophageal fistula as a neonate but remained ventilator dependent for months with left main bronchus (LMB) compression. Separation from the ventilator was eventually achieved, after a failed aortopexy, with a $20 \times 6 \mathrm{~mm}$ Palmaz metallic stent inserted into the LMB. This permitted discharge home for 2 years but she gradually outgrew the neonatal stent, which had become embedded within the LMB.

She represented with recurrent chest infections and left lung collapse, thus necessitating removal and replacement of the LMB stent. The patient also had aortic valve stenosis and required corrective open heart surgery. As she was undergoing bypass, it was decided to remove the embedded bronchial stent during the valve repair.

During bypass surgery, a small bronchial incision was made through which the embedded stent was removed. Due to heparinisation, the procedure was complicated by significant mucosal bleeding into the LMB, which filled the left lung with blood. Postprocedure, the left lung remained opacified on $\mathrm{X}$-ray (figure $1 \mathrm{~A}$ ). The PICU team were unable to open the lung despite physiotherapy, saline lavage, bronchoscopy and high-pressure dual lung ventilation. However, she has been transferred to the ward self-ventilating in air and her last chest X-ray showed reinflation of the left lung, how was this achieved given that all conventional methods failed?

AD: First, we had to restore patency to LMB, which remained collapsed and malacic. We did this using a novel customised biodegradable airway stent.

SB: What are the characteristics of the biodegradable stent that makes it superior to the bare metal stent?

GM: The stent is made of polydiaxonone suture material woven into a mesh and is called an Ella stent. It dissolves by enzyme degradation over 12 weeks and should provide enough time for the patient to recover from bypass surgery. The Ella stent has enough strength to support the airway temporarily but is not as strong as the metal stent. The Ella stent maintained airway patency, while a customised hybrid (metal and silicon) stent was developed.
When she initially presented, the only option to relieve her airway obstruction in the UK was the use of the bare metal stent. Bare metal stents have been used to overcome airway obstruction in children but are complicated by the increased risk of embedment and limited adaptation for the growth of the airway. ${ }^{1}$ Furthermore, they are considered permanent because the removal is risky. Replacement with a bare metal stent would have caused more complication and the biodegradable stent served a temporary bridging measure, allowing airway patency and clearing of the debris and blood that had engorged the lung after the bypass surgery.

SB: Why was a metallic bronchial stent used initially when she first presented and were there any other options?

AD: Biodegradable stents have only recently been introduced in the last few years and remain experimental. In that era, the only options available for stenting a bronchus were metallic stents. Five years ago, before biodegradable stents were available, metallic stents were the last resort when all other options had been exhausted. Complications such as erosion and stent embedment are well recognised with metallic stents ${ }^{1}$; however, if that is the only option to separate a patient from a ventilator after surgery fails, then it was worth taking. Even ventilation via a tracheostomy with a blocked bronchus does not help and restoring bronchial patency is essential. The patient also experienced left lung collapse, which ultimately leads to bronchiectasis.

SB: Are these stents safe for use in the paediatric cohort and can the stent last longer than 12 weeks?

GM: Biodegradable airway stents have been used in the paediatric population as a last resort, when other methods have failed. ${ }^{2}$

Unfortunately, the Ella stent is the only available biodegradable airway stent for use in UK. The Ella stent was originally designed as a temporary oesophagheal stent for strictures in adults; however, we use it specifically in the paediatric airway as it is safe and there is no risk of embedment. Because of enzymatic degradation, its half-life cannot be prolonged. Its use remains experimental and in future more durable materials will be available, for example, biodegradable metal alloys.

SB: Once the stent is in place, how are the debris and blood removed from the lung?

AD: After successfully stenting the LMB, we used an inert, organofluorine compound called perfluorocarbon (PFC) that has previously been used for liquid ventilation. ${ }^{3}$ PFC is a biologically inert, highly stable organic hydrocarbon that is unique in 

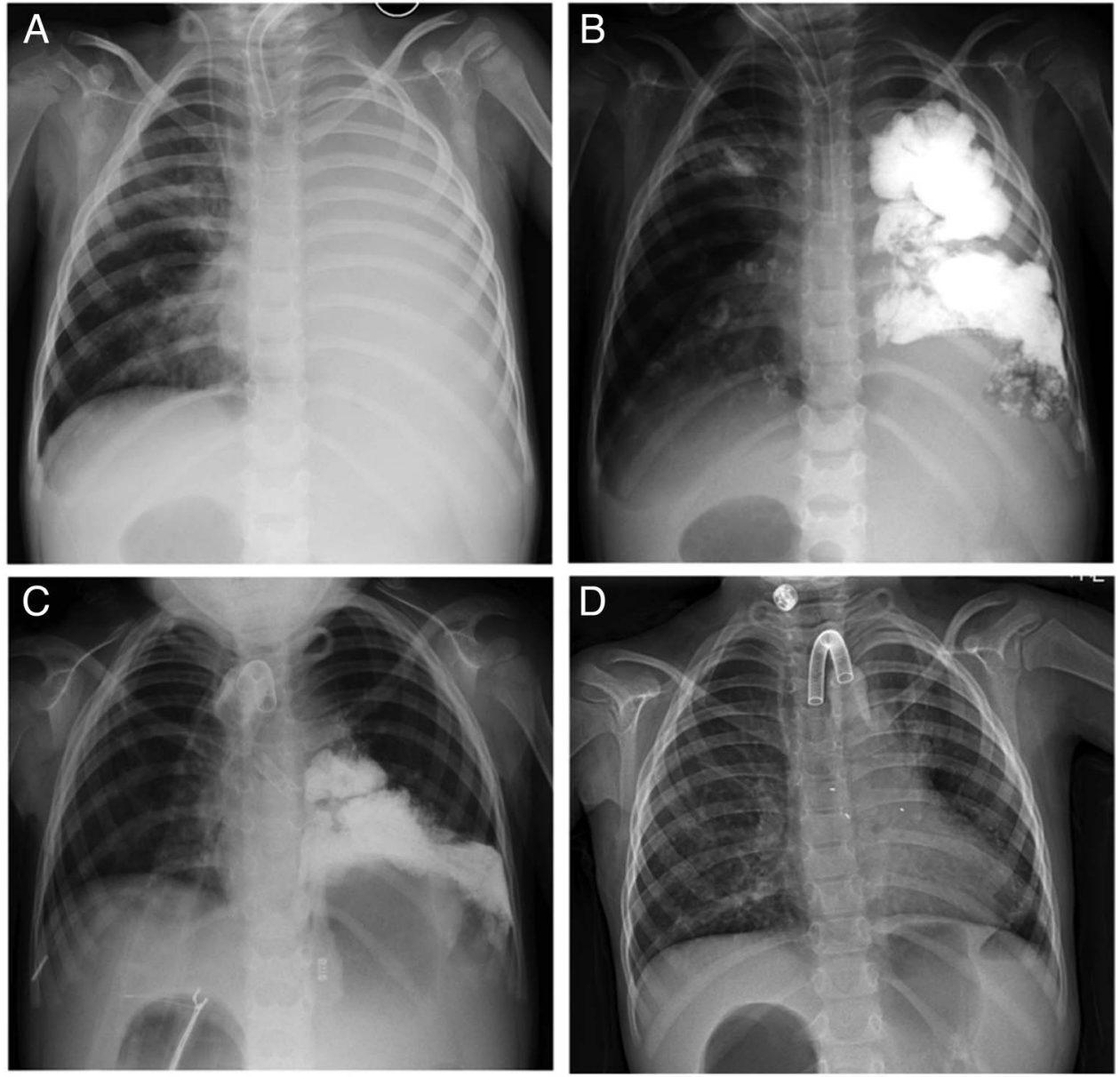

Figure 1 (A) Collapsed left lung immediately following cardiac bypass and surgical removal of left main bronchus (LMB) stent; (B) perfluorocarbon (PFC) instillation into the left lung, (C) partial resolution of PFC 1 week after instillation. Temporary covered metallic stent is visible in LMB and (D) full re-expansion of left lung with evaporation of PFC after 1 month. Metallic gold tips of biodegradable stent visible at start and end of LMB.

its high oxygen-carrying capacity and high density. It is readily dissolved in the presence of air and, in a well-ventilated lung, can evaporate in a few hours. PFC disperses into the bronchial tree and recruits alveoli. In areas that are adequately ventilated, PFC evaporates. In areas of atelectasis, because of its high density, secretions and debris (including blood and mucus) float above the PFC and can be removed bronchoscopically.

PFC is available in a radiopaque form, thus when instilled conventional radiography can be used to monitor the progress of lung clearing and sequential targeted bronchoscopies can be used to clear the lung using suction and lavage of the atelectatic segments.

SB: Is PFC licensed for this use and has it undergone a randomised control trial?

AD: PFC has been used in anaesthesia since 1969. They were first used as an alternative means of oxygen delivery and in the 1990s there was interest in using PFC as an alternative to blood. However, the clinical trials that have been carried out thus far using PFC for partial liquid ventilation have not been promising. A phase II trial is being carried out, the results of which are expected later this year. PFC is currently used in vitreoretinal surgery $^{4}$ and as an alternative means of drug delivery in chemotherapy. ${ }^{5}$

SB: How is the PFC instilled and how much is given?

AD: PFC was instilled via a bronchoscope directed down the LMB over approximately $5 \mathrm{~min}$. A target volume of approximately $2 \mathrm{~mL} / \mathrm{kg}$ is used. This is usually to fill the lung until a fluid level is visualised in the bronchus.

SB: So, when do you suction the secretions and debris out and how long does it take to evaporate?

AD: PFC evaporates over hours when exposed to air if the lung was ventilating well. If the PFC is retained, it indicates an ongoing obstruction or plugging of that segment. In this case, we achieved a rapid improvement in the left lung atelectasis in the first 24 hours and airway pressures were reduced from peak pressures of $30 \mathrm{cmH}_{2} \mathrm{O}$ to below $20 \mathrm{cmH}_{2} \mathrm{O}$. However, it still took days to clear some of the smaller lower lobe subsegmental bronchi.

SB: I note from the X-rays that there was a sequential improvement, how was this achieved?

AD: To achieve the final result, the patient had three sequential bronchoscopies undertaken over the course of a week and complemented this with aggressive lavage and physiotherapy of the blocked segments, particularly the left lower lobe.

During bronchoscopy, large amounts of blood-stained secretions and mucus were suctioned. Afterwards, the physiotherapy and imaging were repeated. Sequential imaging identified specific areas of collapse that were targeted with greater accuracy on subsequent bronchoscopy. Following this, ventilation was rapidly weaned over days back to the patient's usual nocturnal ventilator support (pressures 15/5) via the tracheostomy. The patient was active and mobile, self-ventilating via the tracheostomy during the day. 
SB: What is the advantage of PFC over the more conventional radiocontrast agents, such as Omnipaque?

$\mathrm{AD}$ : PFC is inert and does not cause airway irritation and has high gas-carrying capabilities like conventional radiocontrast agents. Furthermore, it does not have the risk of causing anaphylactic reaction or impact on renal function, because its systemic absorption from the alveoli is limited. Furthermore, because of its oxygen-carrying capacity, it served two functions in our patient: ventilation and visualisation of the blocked areas of the left lung.

SB: The last X-ray still shows a patchy distribution of PFC, how will this be resolved?

AD: This will evaporate with time as the patient's ventilation gradually improves over time and she has better aeration of the left lung. PFC is relatively inert and, over the long term, may be taken up by macrophages and removed. ${ }^{4}$

Contributors $A D, G M, S T$ and $A N$ were involved in clinical care of the patient; $S B$, $A D$ and GM wrote the manuscript and AN produced the figure.
Competing interests None declared.

Patient consent Obtained.

Provenance and peer review Not commissioned; externally peer reviewed.

\section{REFERENCES}

1 Valerie EP, Durrant AC, Forte $V$, et al. A decade of using intraluminal tracheal/bronchial stents in the management of tracheomalacia and/or bronchomalacia: is it better than aortopexy? J Pediatr Surg 2005;40:904-7; discussion 907.

2 Vondrys D, Elliott MJ, McLaren CA, et al. First experience with biodegradable airway stents in children. Ann Thorac Surg 2011;92:1870-4.

3 Leach $C L$, Greenspan JS, Rubenstein D, et al. Partial liquid ventilation with perflubron in premature infants with severe respiratory distress syndrome. $N$ Eng J Med 1996;335:761-7.

4 Chang S. Low viscosity liquid fluorochemicals in vitreous surgery. Am J Opthalmology 1987; 103:38-43.

5 Ma M, Xu H, Chen $\mathrm{H}$, et al. A drug-perfluorocarbon nanoemulsion with an ultrathin silica coating for the synergistic effect of chemotherapy and ablation by high-intensity focused ultrasound. Adv Matter 2014;26:7378-85. 